\title{
INFINITESIMAL APPROACH TO THE CRYSTALLOGRAPHY OF MARTENSITIC TRANSFORMATION: APPLICATION TO Ni-Ti
}

\author{
Yuanchang Liang, Hiroyuki Kato*, Minoru Taya and T. Mori \\ Department of Mechanical Engineering, University of Washington, Seattle, WA 98195
}

(Received April 12, 2000)

(Accepted May 9, 2000)

Keywords: Twinned martensite; Martensite plate morphology; Infinitesimal theory

\section{Introduction}

To determine flow curves of a material, whose plastic deformation is produced by martensitic transformation, transformation strains of a martensite plate must be characterized [1-3]. Most studies along this line adopt results obtained by the phenomenological crystallographic theory of martensitic transformation [4,5]. The theory is based on finite deformation analysis, which needs involved computations. Solutions with analytical forms have been obtained in a few limited cases [6]. However, if we take an infinitesimal deformation theory, the calculation becomes simplified. Moreover, the infinitesimal theory offers simple and clear pictures of the computations. The present study demonstrates this point. Also, if stress analysis is involved as in polycrystal transformation plasticity [1-3], infinitesimal theory is rather adequate, since stress analysis is usually based on infinitesimal theory. That is, consistency with using Hooke's law is observed.

\section{Analysis}

It has been shown that when an infinitely extended plate-like region, the plate plane (habit plane) being perpendicular to the $\mathrm{z}$-axis, has the eigenstrain satisfying the condition

$$
\epsilon_{x x}^{*}=\epsilon_{y y}^{*}=\epsilon_{x y}^{*}=0,
$$

neither stress nor strain energy is produced [7]. This result is valid, whether or not anisotropy in elastic constants exists, and regardless of the elastic constants of the martensite differing from those of the matrix. The transformation strain of a Bain correspondence variant (BCV) cannot, in a usual case, achieve condition (1) for any possible direction of the plate plane. Thus, a standard way is to seek the condition, by forming a combined variant (twinned martensite in most cases), whose average transformation strain is written as

$$
\overline{\epsilon_{i j}^{T}}=f \epsilon_{i j}^{T}(M)+(1-f) \epsilon_{i j}^{T}(N) .
$$

Here, $\epsilon_{i j}^{T}(M)$ stands for the transformation strain of the M-th BCV. $f$ is the fraction of this variant in the combined variant to make condition (1) satisfied. One has also to determine the habit plane normal at

\footnotetext{
* Permanent address: Department of Engineering Physics and Mechanics, Kyoto University, Kyoto 606-8501, Japan. 
the same time. In addition, since no clear criterion to pick up an acceptable combination of the two $\mathrm{BCV}$ s to form (2) has been given yet, some trials are needed in the computation. The present study will show how the approach using infinitesimal theory simplifies computational processes.

Condition (1) is equivalently written in terms of the principal strains, $\epsilon_{1}, \epsilon_{2}$ and $\epsilon_{3}$ : One of them, say $\epsilon_{3}$, is zero and the product of the other two is not positive. That is,

$$
\begin{gathered}
\epsilon_{3}=0, \\
\epsilon_{1} \epsilon_{2} \leq 0 .
\end{gathered}
$$

Condition (3) with (4) is equivalent to condition (1). Using the two conditions, we can find a combined variant more economically than the methods used before $[8,9]$; the methods which examined elastic energy of a combined variant in terms of $f$, the orientation of the plate (habit) plane etc. That is, the average strain is, first, more easily found without considering a possible habit plane, the orientation of which is later determined by geometrical analysis.

Let us consider the situation that the transformation strain $\epsilon_{i j}^{T}$ is written in the orthogonal coordinate system (X) in the parent phase. The determinant of the strain is invariant, so that condition (3) is expressed as

$$
\operatorname{det}\left(\overline{\epsilon_{i j}^{T}}\right)=0
$$

Nothing that $\overline{\epsilon_{i i}^{T}}$ and $\overline{\epsilon_{i j}^{T}} \overline{\epsilon_{i j}^{T}}$ are invariant, we can rewrite the condition (4) as

$$
\overline{\epsilon_{i i}^{T}} \overline{\epsilon_{j j}^{T}}-\overline{\epsilon_{i j}^{T}} \overline{\epsilon_{i j}^{T}} \leq 0 .
$$

The next task is to determine $f$, with which a proper combination of two BCVs to form the combined variant of (2) satisfies conditions (5) and (6). When there are many BCVs, it is better to decrease the number of the possible combinations from the beginning. The following is one method to achieve this process.

The presence of a stress free interface between the two possible BCVs also means that the conditions similar to (5) and (6) must be satisfied. It is written as a function of the difference, $\delta \epsilon_{i j}^{T}$, of the transformation strain between the variants.

$$
\delta \epsilon_{i j}^{T}=\epsilon_{i j}^{T}(N)-\epsilon_{i j}^{T}(M) . \quad \text { in X }
$$

The condition corresponding to (5)

$$
\operatorname{det}\left(\delta \epsilon_{i j}^{T}\right)=0
$$

must first be satisfied. Then, the condition corresponding to (6)

$$
\delta \epsilon_{i i}^{T} \delta \epsilon_{j j}^{T}-\delta \epsilon_{i j}^{T} \delta \epsilon_{i j}^{T} \leq 0
$$

is automatically satisfied. Note that the first term in the left hand side is zero, because $\delta \epsilon_{i i}^{T}$ is the difference of the volume strain, which is zero. $\delta \epsilon_{i j}^{T} \delta \epsilon_{i j}^{T}$ is the positive definite, so that (9) is always valid, as long as (8) is observed. These properties of the possible combination of the BCVs save some extra computations.

Equation (5) might appear a cubic equation of $f$. However, it is quadratic, since (8) is imposed on $\epsilon_{i j}^{T}(M)$ and $\epsilon_{i j}^{T}(N)$. When (5) is expanded, the coefficient for the cubic term of $f$ becomes the determinant of $\delta \epsilon_{i j}^{T}$, which is set zero from the beginning. Thus, the solution of $f$ to satisfy (5) can be obtained elementally. It appears that further simplification cannot be achieved, as we have found in an example of the martensitic transformation in Ni-Ti. 


\section{Martensite Plate in Ni-Ti}

\subsection{Combined Variant and Average Transformation Strain}

The lattice parameter of the cubic parent phase is $0.3015 \mathrm{~nm}$ and those of the monoclinic martensite phase are $\mathrm{a}_{\mathrm{M}}=0.2889 \mathrm{~nm}, \mathrm{~b}_{\mathrm{M}}=0.4120 \mathrm{~nm}, \mathrm{c}_{\mathrm{M}}=0.4622 \mathrm{~nm}$ and $\beta=96.8^{\circ}$ [10]. The Bain correspondence gives a $\mathrm{BCV}$ the transformation strain of

$$
\epsilon_{i j}^{T}(3-1)=\left(\begin{array}{ccc}
a & c & -d \\
c & a & -d \\
-d & -d & b
\end{array}\right) \quad \text { in } \mathrm{X}
$$

where $a=0.02132, b=-0.04179, c=0.05505$ and $d=0.04216$. Here, the coordinate axes are parallel to [001], [010] and [001] in the parent phase, bases of the X system. We are aware that only three digits in (10a) are meaningful. However, for computational purposes, we will use four digits. This applies to all the following calculations. Three more similar and equivalent variants exist and their transformation strains are

$$
\begin{gathered}
\epsilon_{i j}^{T}(3-2)=\left(\begin{array}{lll}
a & c & d \\
c & a & d \\
d & d & b
\end{array}\right) \text { in } \mathrm{X} \\
\epsilon_{i j}^{T}(3-3)=\left(\begin{array}{ccc}
a & -c & d \\
-c & a & -d \\
d & -d & b
\end{array}\right) \text { in } \mathrm{X} \\
\epsilon_{i j}^{T}(3-4)=\left(\begin{array}{ccc}
a & -c & -d \\
-c & a & d \\
-d & d & b
\end{array}\right) . \text { in } \mathrm{X}
\end{gathered}
$$

Here, the first number in the parenthesis denotes the $\mathrm{BCV}$, whose $\mathrm{c}_{\mathrm{M}^{-}}$-axis corresponds to [001] of the parent phase. By cyclic changes of the direction, the transformation strain of the other eight BCVs are similarly written. That is, there are $12 \mathrm{BCVs}$ in total. Among the 66 combinations of these BCVs, 42 combinations satisfy condition (8).

Also, out of the above 42 combinations, 18 combinations are unsatisfactory, since the root for $f$ of (5) does not become real. The remaining 24 are classified into two groups, A and B. A combined variant in group $\mathrm{A}$ is, for example, formed by $(3-1)$ with $(3-3)$ or $(3-4)$. The symmetry gives total of 12 possible combinations belonging to this group. $f$ is written as

$$
\mathrm{f}=\frac{1}{2} \pm \frac{1}{2} \sqrt{\frac{\mathrm{a}\left(\mathrm{ab}-\mathrm{d}^{2}\right)}{\mathrm{bc}^{2}-\mathrm{d}^{2}(-\mathrm{a}+2 \mathrm{c})}}=0.2764 \text { or } 0.7236
$$

A combined variant in group B is, for example, formed by the combination of (3-1) with (1-1) or (2-1). There are, in total, 12 such combinations. The analytical form of $f$ for this group is so lengthy that only the numerical result is given below.

$$
f=0.3102 \text { or } 0.6898 \text {. }
$$

Since the two roots of (11A) and (11B) produce equivalent but different average transformation strains expressed in the identical coordinate systems, each group has 24 equivalent combined variants.

The average transformation strain of a variant in group A is written as 


$$
\overline{\epsilon_{i j}^{T}}=\left(\begin{array}{ccc}
0.02132 & 0.01885 & 0.02462 \\
0.01885 & -0.04179 & 0.04216 \\
0.02462 & 0.04216 & 0.02132
\end{array}\right), \quad \text { in } \mathrm{X}
$$

and that for a variant in group B is

$$
\overline{\epsilon_{i j}^{T}}=\left(\begin{array}{ccc}
0.02132 & 0.01200 & 0.02490 \\
0.01200 & -0.02221 & 0.04216 \\
0.02490 & 0.04216 & 0.001738
\end{array}\right) . \quad \text { in } \mathrm{X}
$$

These are, of course, expressed in analytical form in terms of a, b, c and d, but becomes too lengthy. Thus, (12A) and (12B) are written numerically.

\subsection{Habit Plane, Normal Strain and Shear Direction of a Combined Variant}

After finding the principal strains and corresponding principal directions of a combined variant, it is straightforward to determine the habit plane normal and shear direction.

(The normal strain is equal to the volume strain, which is equal to that of a BCV.) For example, (12A) is converted to

$$
\overline{\epsilon_{i j}^{T}}(\operatorname{diag})=\left(\epsilon_{1}, \epsilon_{2}, 0\right)
$$

where $\epsilon_{1}=0.06419, \epsilon_{2}=-0.06335$. The principal direction for $\epsilon_{1}$ is $[0.5813,0.3879,0.7153]$ and that for $\epsilon_{2}$ is $[0.0769,-0.9012,0.4265]$. The other direction is $[0.8101,-0.1931,-0.5536]$. From these results, we can find two possible habit planes. The tensile strain along the last direction is zero. The other direction, along which the tensile strain is also zero, can be found using the property of the representative quadratic [7]. These two directions correspond to the $\mathrm{X}_{1}$ - and $\mathrm{X}_{2}$ - directions defined in (1). The direction, normal to the two directions, is the habit plane normal, $\mathbf{n}$. When expressed in the orthogonal system defined by the three principal directions, the normal can have two directions written, respectively, as

$$
\mathbf{n}=\left\lfloor\sqrt{\epsilon_{1} /\left(\epsilon_{1}-\epsilon_{2}\right)}, \sqrt{-\epsilon_{2} /\left(\epsilon_{1}-\epsilon_{2}\right)}, 0\right\rfloor
$$

and

$$
\mathbf{n}=\left\lfloor\sqrt{\epsilon_{1} /\left(\epsilon_{1}-\epsilon_{2}\right)},-\sqrt{-\epsilon_{2} /\left(\epsilon_{1}-\epsilon_{2}\right)}, 0\right\rfloor
$$

These are easily expressed in the $\mathrm{X}$ system. That is, the normal direction corresponding to (14) is $[0.3584,-0.2069,0.9104]$ in the orthogonal system defined in the parent phase. That corresponding to (15) is [-0.4664, 0.8080, 0.3600].

The corresponding parameters for $(11 \mathrm{~B})$ are listed below. $\epsilon_{1}=0.05534$ with the principal direction of $[0.6257,0.4451,0.6406]$ and $\epsilon_{2}=-0.05450$ with that of $[0.0802,0.7802,-0.6203]$. The principal direction for the zero principal strain is $[0.7759,-0.4396,-0.4525]$. Of course, the normal of the habit plane has the same form as (14) and (15). One is expressed in the orthogonal system in the parent phase as $[0.2336,0.3876,0.8917]$ and the other $[-0.8654,0.5006,0.0178]$.

The direction $\lambda_{i}$ of the total deformation, corresponding to the displacement vector in the shape deformation examined in the phenomenological theory, is now determined. Consider a tensile stress with a unit strength along a unit vector $\mathrm{m}_{\mathrm{i}}$. The work supplied to the formation of a martensite plate (per unit volume) is

$$
W=m_{i} m_{j} \overline{\epsilon_{i j}^{T}}
$$


When $m_{i}$ is equal to $\lambda_{i}, W$ becomes maximum. That is, the direction to calculate is to maximize (18) under the condition of

$$
m_{i} m_{i}=1 .
$$

The process is exactly the same as that to obtain one of the principal directions of $\overline{\epsilon_{i j}^{T}}$, the direction corresponding to the largest principal strain. This has already been obtained.

Since the normal direction of the plate and the normal strain have already been determined, the magnitude of shear and its direction are determined in a straight forward manner. For example, the shear direction examined in $(13 \mathrm{~A})$ is $[-0.4641,0.8067,0.3660]$ and the magnitude of the shear is 0.126 .

\subsection{Interface Plane between Two BCVs to Form a Combined Variant}

The method to determine the interface between two BCVs is the same as that used for the habit plane determination, except that (7) is used instead of (2). The combination of (3-1) and (3-3) $(f=0.2764)$ can have two interface planes. The normal vector of one is written as

$$
\nu=[1,0,0] \text { in } \mathrm{X}
$$

and that of the other is

$$
\nu=[0,1,-d / c]=[0,1,-0.8221] . \quad \text { in } \mathrm{X}
$$

Using the lattice correspondence for the BCV of (10a),

$$
(h k \lambda)_{\mathrm{M}}=(h k \lambda)_{\mathrm{P}}\left(\begin{array}{ccc}
1 & 1 & 0 \\
-1 & 1 & 0 \\
0 & 0 & 1
\end{array}\right)
$$

for the planes, the interface given by $(18)$ is found to correspond to $(1,1,0)$ of the martensite. (Here, $(h$ $k \lambda)_{\mathrm{M}}$ is the index of a plane in the martensite and $(h k \lambda)_{\mathrm{P}}$ is that in the parent phase.) The interface given by (19) is irrational, $(1,-1,0.7657)$ in the martensite.

The combination by $(3-1)$ and $(1-1)(f=0.3102)$ can have an interface normal to

$$
\nu=[1,0,-1] \text { in } \mathrm{X}
$$

or

$$
\nu=[1,3.081,1] \text { in } \mathrm{X}
$$

in the parent phase. These are, respectively, expressed in the martensite lattice as $(1,1,-1)$ and $(0.5098$, $1,0.2450)$ plane. The interfaces of (18) and (21) are twinned planes in the martensite lattice.

\subsection{Comparison with Phenomenological Theory Calculations}

Here, the paper by Knowles and Smith [11] and that by Matsumoto, Miyazaki, Otsuka and Tamura [12], the calculations based on the phenomenological theory, are referred to. The former reported two values, 0.2710 and 0.3201 , corresponding to our $f=0.2764$ and $f=0.3102$. The difference is not much. The latter dismissed the case corresponding to 0.3201 .

The habit plane reported by Matsumoto et al in $f=0.2710$ is $(0.8889,0.4044,0.2152)$. Our value is $(0.9104,0.3584,0.2069)$. The difference is only $3^{\circ}$. The habit plane corresponding to our $(0.8080$, $0.4664,0.3600), f=0.3102$, is possibly that for the (-) solution by Matsumoto et al for $f=0.2710$; 
$(0.7712,0.5136,0.3762)$. These directions differ by $4^{\circ}$. The amount of the shear, 0.126 , calculated in our approach, compares well with that given by Matsumoto et al, 0.131 .

The rational interface plane, $(1,1,0)$ for $f=0.2710$ and $(1,1,-1)$ for $f=0.3102$, were also reported by both groups. The former is the type II twin plane and the latter the type I twin plane. However, it appears that neither of the two groups examined the other two possible interfaces, $(1,1,0.7657)$ for $f=0.2764$ and $(0.5099,1,0.2450)$ for $f=0.3102$. Since these are irrational, the dismissal of these planes might be justified.

\section{Concluding Remarks}

One advantage of using the infinitesimal approach is easily to find a possible combination of two BCVs. When the number of BCVs is small as in the case of Fe-based alloys, one can choose the combinations readily. However, when the number is large, the determination of the possible combinations is not straightforward. We have not seen an easy and logical method to apply to this subject yet. The present method offers one easy method.

In many respects, the analysis based on infinitesimal theory is simple. We have demonstrated this point by analyzing the fraction of a BCV and the habit plane of a Ni-Ti martensite plate. Also, note that the interface plane between two BCVs forming a combined variant is automatically found. Further, the calculations certainly indicate that the infinitesimal analysis gives almost the same results as the phenomenological theory.

The present study has also completed the analysis of the deformation of a group B combined variant ( $f=0.3012$ ), which neither Knowles and Smith nor Matsumoto, Miyazaki, Otsuka and Tamura reported. By taking into account the average transformation strains of both groups $\mathrm{A}$ and $\mathrm{B}$, we have found that group B cannot be ignored, when the preferential formation of a particular variant is examined under stress. For example, if a single crystal is compressed, group B variants are formed with less stress than group A variants, when the loading axis is near the [101]-[111] boundary of the standard triangle. Since there is no decisive factor to dismiss group B, the transformation under stress must be examined, by counting the roles of the variants in this group together with those in group A.

\section{$\underline{\text { Acknowledgment }}$}

The present study was supported by New Energy and Industrial Technology Development Organization, Japan.

\section{$\underline{\text { References }}$}

1. N. Ono and H. Shimanuki, Scripta Metall. Mater. 24, 2269 (1990).

2. K. Gall, H. Sehitoglu, Y. I. Chumlyakov, and I. V. Kireeva, Acta Metall. 47, 1203 (1999).

3. Y. Linag, H. Kato, M. Taya, and T. Mori, to be submitted.

4. J. S. Bowels and M. S. Mackenzie, Acta Metall. 2, 129 (1955).

5. D. S. Lieberman, M. S. Wechsler, and T. A. Read, J. Appl. Phys. 26, 473 (1955).

6. H. Kato, Scripta Mater. 38, 1125 (1999), ibid, 39, 999 (1999).

7. T. Mura, Micromechanics of Defects in Solids, p. 143, Martinus Nijhoff (1987).

8. M. Kato and H.-R. Pak, Phys. Stat. Sol. (b). 130, 421 (1985).

9. H. Ledbetter and M. Dunn, in International Conference on Displacive Phase Transformations and Their Applications in Materials Science, p. 341, TMS (1998).

10. K. Otsuka, T. Sawamura, and K. Shimizu, Phys. Stat. Sol. 5, 457 (1971).

11. K. M. Knowles and D. A. Smith, Acta Metall. 29, 101 (1981).

12. O. Matsumoto, S. Miyazaki, K. Otsuka, and H. Tamura, Acta Metall. 35, 2137 (1987). 